\title{
The Algorithm of the Clutter and Interference Deletion Based on the Image Technique of the Group Segmentation
}

\author{
Haipeng Wang, Shuyi Jia and Tiantian Tang \\ Department of Electronic and Information Engineering, Naval Aeronautical and Astronautical University, \\ Yantai Shandong, 264001
}

\begin{abstract}
To resolve the multi-sensor tracking problem of the targets in a non-maneuvering formation with the complicated background, based on the image technique of the group segmentation, the deletion models of the cloud-rain clutter and the banded interference were established, and the validity was analyzed with the simulation data and the actual data.
\end{abstract}

Keywords- the group segmentation; cloud-rain clutter; banded interference; non-maneuvering formation

\section{INTRODUCTION}

Track maintenance is the core content of the target tracking process. Compared with the traditional multi-target, track maintenance of the targets in formation is more complicated, and the existing algorithms of formation targets tracking are mostly based on the overall formation tracking without considering the problem of targets tracking in a formation; while the tracking environment of track maintenance of the targets in a formation is relatively single in some algorithms, which is difficult to apply to the complex background such as cloud-rain clutter [1-4], banded interference[5-9] and so on. To solve this problem, the characteristics of targets in a formation is mainly discussed in this paper. These formation targets are with centralized multi-sensor system in complex environment such as cloud-rain clutter, banded interference and so on. It is researched that how to maximize the detection of adverse effects of cloud-rain clutter and banded interference under the premise of not affecting the true echo of the targets in a formation. Moreover the deletion models of the cloud-rain clutter and the banded interference are established to realize clutter pretreatment of formation target tracking. Thus the foundation is laid on the accurate tracking of the targets in a formation.

\section{SYSTEM DEFINITION}

The centralized multi-sensor tracking problem of the targets in a formation is that Ns sensors in the clutter are used to report fusion center measurement for achieving state update of each target in a formation. It is assumed that each measurement most likely comes from a target, each target has a maximum real echo value in each time, and some sensors may not provide measurement in each interval. The dynamic equation of the system is set that

$$
\boldsymbol{X}^{t}(k+1)=\boldsymbol{F}(k) \boldsymbol{X}^{t}(k)+\boldsymbol{\Gamma}(k) \boldsymbol{V}^{t}(k)
$$

$$
k=1,2, \mathrm{~L} ; t=1,2 \mathrm{~L} T_{g}
$$

Where $\boldsymbol{X}^{t}(k+1) \in \boldsymbol{R}^{n}$ is the global state vector of the target at $\mathrm{k}+1$ time, $\boldsymbol{F}(k) \in \boldsymbol{R}^{n}$ is the state transition matrix, $T_{g}$ is the number of targets in $g$ formation, $\boldsymbol{\Gamma}(k) \in \boldsymbol{R}^{n, h}$ is the noise distribution matrix, and $\boldsymbol{V}^{t}(k)$ is the fourier white noise sequence, also $E\left[\boldsymbol{V}^{t}(k)\right]=0, E\left[\boldsymbol{V}^{t}(k) \boldsymbol{V}^{t}(k)^{\mathrm{T}}\right]=\boldsymbol{Q}^{t}(k)$.

$m_{k_{i}}\left(i=1,2, \ldots, N_{s}\right)$ is set to the number of measurements from the sensor $i$ at $\mathrm{K}$ time. In the multi-sensor environment, the measurement equation is expressed as:

$$
\boldsymbol{Z}_{l}^{i}(k)=\boldsymbol{H}_{i}(k) \boldsymbol{X}^{t}(k)+\boldsymbol{W}_{l}^{i}(k), t=1,2, \ldots, T ; l=1,2, \ldots, m_{k_{i}}
$$

Where the measurement $\boldsymbol{Z}_{l}^{i}(k)$ is the $l$ measurement from the sensor $i$ at $\mathrm{k}$ time, $\boldsymbol{H}_{i}(k)$ is the measurement matrix of the sensor $i, \boldsymbol{W}_{l}^{i}(k)$ is the zero mean Gaussian noise vector with known covariance and statistically independent of all other noise vector, $T$ is the number of targets. So the comprehensive observation vector obtained in fusion center at $k$ time is

$$
\boldsymbol{Z}(k)=\left\{\boldsymbol{Z}_{i}^{s}(k)\right\} \quad i=1, \mathrm{~L}, m_{s} ; s=1, \mathrm{~L}, N_{s}
$$

And it is assumed that the measurement error among the sensors is statistically independent.

\section{The Deletion Models OF THE ClOUd-RAin ClutTer AND THE BANDED INTERFERENCE}

When there are the cloud-rain clutter and the banded interference in the sensor detection area, the false measurement caused by the cloud-rain clutter and the banded interference should be eliminated before tracking for targets in a formation. Therefore the detection model of the cloud-rain clutter and the banded interference detection model were respectively established in this section based on the idea of the image technique of group segmentation[10]. 


\section{A. The Deletion Model of the Cloud-Rain Clutter}

When the sensor lifts off or its detection area is in the cloud-rains and rain weather, it will face strong cloud-rain clutter. Especially when the targets are in the cloud-rain clutter zone, the detection performance of the sensor will be seriously affected. High density of measurement points within a certain area are assumed as the cloud-rain clutter in the level of data processing. It would be in the presence of multiple detection area to form a plurality of false track, which is not conducive to the formation of the overall situation of the space target. Therefore the detection model of the cloud-rain clutter is established to exclude the false measurement. Specifically for the following four steps:

1) The Establishment of Measurement Area Matrix

All measurement in $\boldsymbol{Z}(k)$ is transformed to the coordinate system of the fusion center. And get a set of the measured maximum and minimum values in the $x, y$ direction. The set is $\boldsymbol{E}_{x y}(k)=\left[z_{\text {max }}^{x}(k), \boldsymbol{z}_{\text {min }}^{x}(k), z_{\text {max }}^{y}(k), z_{\text {min }}^{y}(k)\right]^{T}$. The rectangle A (as shown in Figure 1) is constructed based on $\boldsymbol{E}_{x y}(k)$, and will be a split into multiple small area according to Figure 2. Thus the measurement area matrix is defined as the rectangular $\mathrm{B}$.

$$
\boldsymbol{R}_{e}(k)=\left\{a_{i j}(k)\right\} \quad i=1,2, \mathrm{~L}, L_{x}(k) ; j=1,2, \mathrm{~L} L_{y}(k)
$$

In the formula, $L_{x}(k)$ and $L_{y}(k)$ are respectively the number of the small area in $x, y$ direction of the rectangular $\mathrm{B}$, whose values depends on the actual tracking system and the task requirements[11].The specific confirmation process is the same as the graphic method.

2) The Establishment of Weight Matrix

The weights are determined based on the number of measured values in $a_{i j}(k)$. The weight matrix of the rectangular $\mathrm{B}$ is defined as

$$
\boldsymbol{W}_{e}(k)=\left\{w_{i j}(k)\right\} \quad i=1,2, \mathrm{~L}, L_{x}(k) ; j=1,2, \mathrm{~L} L_{y}(k)
$$

In the formula, the initial value of $w_{i j}(k)$ is zero. Execute the operation $w_{i j}(k)=w_{i j}(k)+1$, every time there is a measurement to fall into $a_{i j}(k)$ in the process of loop operation to $\boldsymbol{Z}(k)$.

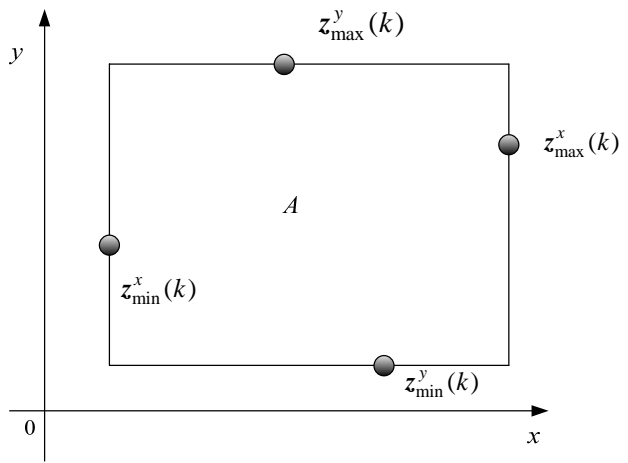

FIGURE I. THE DIAGRAM OF MEASUREMENT AREA

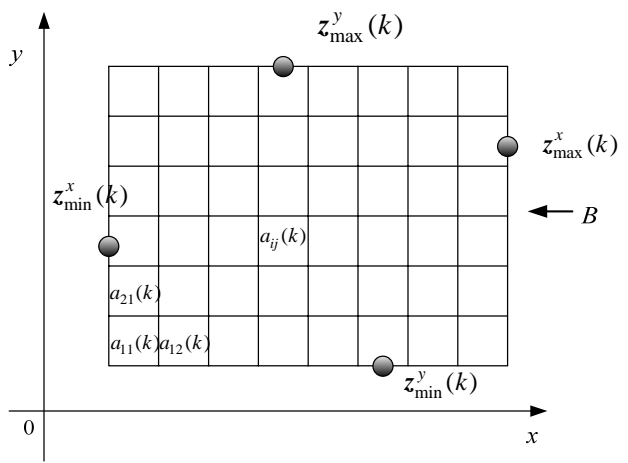

FIGURE II. THE DIAGRAM OF MEASUREMENT AREA MATRIX

3) The Establishment of Evolutionary Weight matrix

To facilitate the distinction between the cloud-rain clutter and the ordinary clutter, the intensive characteristics of the cloud-rain clutter are needed to further enlarge in through the evolution of weight matrix. The evolutionary weights $w_{i j}(k)$ of $a_{i j}(k)$ is defined as $w_{i j}(k)$. The sum of $w_{i j}(k)$ and the weights of its adjacent area are

$$
W_{i j}(k)=\sum w_{\left(i+l_{x}\right)\left(j+l_{y}\right)}(k) \quad l_{x} \in[-1,0,1] ; l_{y} \in[-1,0,1]
$$

Thus the evolutionary weight matrix of the rectangular B is

$$
\hat{W}_{e}(k)=\left\{\hat{w}_{i j}(k)\right\} \quad i=1,2, \mathrm{~L}, L_{x}(k) ; j=1,2, \mathrm{~L} L_{y}(k)
$$

4) The Establishment of Detection Rule

The mean evolutionary weight is defined as

$$
\bar{w}(k)=\frac{1}{\bar{l}} \sum_{i=1}^{L_{x}(k)} \sum_{j=1}^{L_{y}(k)} w_{i j}(k)
$$

In the formula, $\bar{l}$ is the number of small areas where the evolutionary weight is not zero.

Because the intensive degree of cloud-rain clutter is far higher than other targets and clutter, so if 


$$
w_{i j}(k)<b \bar{w}(k)
$$

retain the measurement in $a_{i j}(k)$, otherwise the measurement in $a_{i j}(k)$ will be eliminated as the cloud-rain clutter. In the formula, $b$ is the detection coefficient, and usually $1 \leq b<\frac{w_{\max }(k)}{\bar{w}(k)} \quad\left(\hat{w}_{\max }(k)\right.$ is the maximum evolutionary weight). Its specific value is related to the target environment. In this note, when the formation target is existed in the target environment, the value of $b$ should be relatively large for avoiding the formation measurement removed as cloud-rain clutter.

\section{B. The Deletion Model of the Banded Interference}

Electromagnetic interference is prevalent in the modern battlefield as the important implementation to reduce the detection ability of the sensor. Banded interference is a common mode of disturbance, which assumes the sector measurement density area centered on the sensor. Eliminate its adverse effects in order to form a clear battlefield situation. The specific detection model is the same as the detection model of the cloud-rain clutter, but the following three points must be noted:

(a) The deletion process of banded interference is completed in the local coordinate system of each sensor;

(b) In the deletion models of the banded interference, the detection area is divided into multiple very small sector;

(c) In the deletion models of the banded interference, the weight matrix and the evolutionary weight matrix of the detection area is established in the polar coordinates.

\section{VERIFICATION ANALYSIS}

\section{A. Verification Analysis of the Simulation Data}

Monte Carlo simulation is adopted in this section in order to verify the effectiveness of the algorithm. Figure 3 is the measurement situation map of cloud-rain clutter and banded interference, figure 4 is the measurement situation map of the clutter detection by using the model presented in this section. As shown in figures, the cloud-rain clutter and banded interference can be better detected by the model presented in this section, and at the same time which not has a greater impact on other measurement.

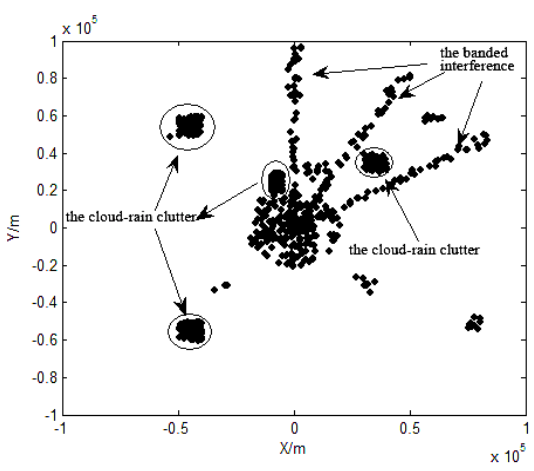

FIGURE III.THE MEASUREMENT SITUATION MAP

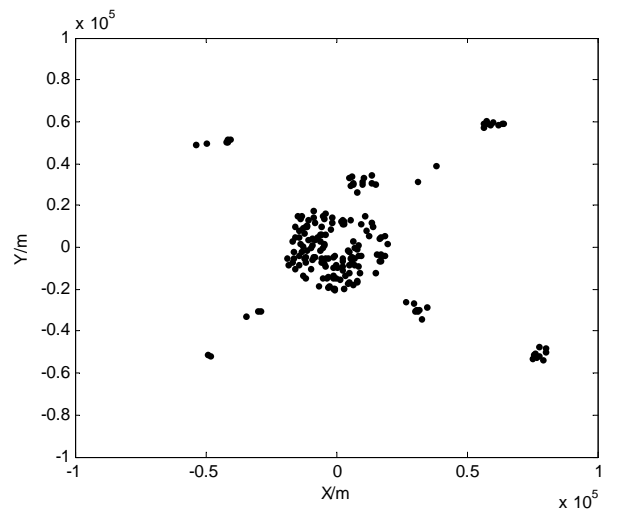

FIGURE IV. THE MEASUREMENT SITUATION MAP OF THE CLUTTER DETECTION

\section{B. Verification Analysis of the Actual Data}

The measured data is adopted in order to further verify the effectiveness of the algorithm presented in this section. Figure 5 is the measurement situation map of the actual data based on "the marine XXX system track fusion competitive advantages ". Figure 6 is the measurement situation map of the results obtained by the model presented in this section As shown in figures, the banded interference is deleted, and the effect is relatively ideal.

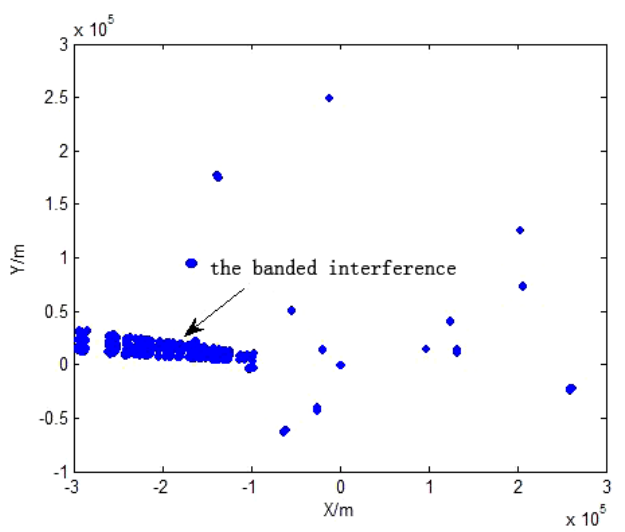

FIGURE V. THE MEASUREMENT SITUATION MAP 


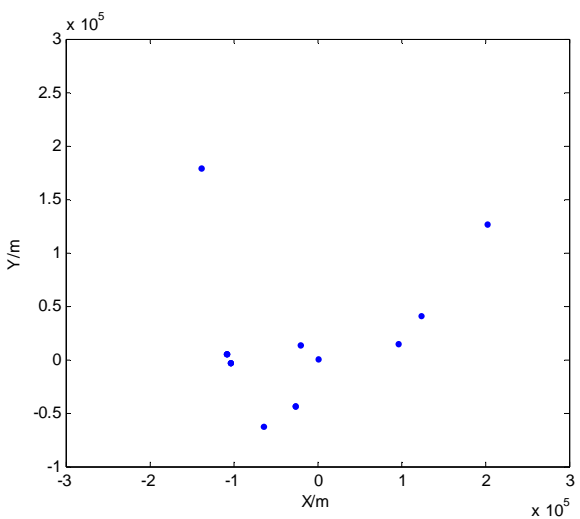

FIGURE VI. THE MEASUREMENT SITUATION MAP OF THE BANDED INTERFERENCE DETECTION

\section{CONCLUSION}

According to the analysis of formation targets characteristic in centralized multi-sensor system in complex environment, based on the image technique of the group segmentation, the deletion models of the cloud-rain clutter and the banded interference are established respectively. Moreover, the validity of the two models is analyzed with the simulation data and the actual data.

\section{REFERENCES}

[1] L. X. Li, Y. Y. Tang, Z. B. Zhu. "Modeling and simulation of rain clutter for tethered areostat borne bistatic radar," Modern Radar,vol.34, no.1,pp.30-36,January 2012.

[2] L. X. Li, Y. Y. Tang, Z. B. Zhu. "Modeling and simulation of rain clutter for tethered areostat borne radar," Radar Science and Technology,vol.8, no.1,pp.15-19,February 2010.

[3] Y. Chen, W. Wu, X. J. Wang ,et al. " Simulation of cloud and rain clutter for VHF band land-air intelligence radar,” Journal of Air Force Radar Academy, vol.24, no.3, pp.163-166, June 2010.

[4] H. H. Benzon, T. Bovith. "Simulation and Prediction of Weather Radar Clutter Using a Wave Propagator on High Resolution NWP Data,” IEEE Transactions on Antennas and Propagation, vol.56, no.12, pp. 3885-3890, 2008.

[5] M. L. Stassen. "Analog and Flexible Digital Suppression of NarrowBanded Interference in UWB,” 2010 17th IEEE Symposium on Communications and Vehicular Technology in the Benelux, vol.11, pp. 24-25, 2010

[6] L. P. Yao, W. D. Zheng, Y. Qian. "A Narrow-banded Interference Suppression Method Based on EEMD for Partial Discharge," Power System Protection and Control, vol.39, no.22, pp. 133-139, 2011.

[7] Z. H. Wang, S. L. Zhou, J. Catipovic. “ Parameterized Cancellation of Partial-Banded Partial-Block-Duration Interference for Underwater Acoustic OFDM,” IEEE Transactions on Signal Processing, vol.60, no.4, pp. 1782-1795, 2012.

[8] H. Wu. "Research on STAP technology of phased array airborne radar in non-uniform clutter environment,” [D]. CS: National Defense Science and Technology University, 2007.

[9] M. Tamaoki, S. Denno, T. Furuno. “Adaptive Multibanded Array for Cancelling Co-Channel Interference and Image-Banded Interference," 2009 IEEE 69th Vehicular Technology Conference, vol.4, pp. 1-4; 26-29, 2009.

[10] H. L. Wang, D. S. Wang, L. S. Tian. "A New Algorithm for Group Tracking,” ICR2001, vol.7, pp. 1159-1163, 2001.

[11] Y. He, J. J. Xiu, J. W. Zhang. Radar data processing and application (Second Edition), BJ: Electronic Industry Press,2009. 